\title{
Massive Pulmonary Embolism Thrombolysis: Early Clinical Markers of Treatment Efficacy
}

\author{
Elie Portnoy ${ }^{1}$ Vibhor Wadhwa ${ }^{2} \quad$ Mariana de Carvalho Barbosa ${ }^{3} \quad$ Clifford R. Weiss ${ }^{1} \quad$ Brian Holly ${ }^{1}$ \\ Mark L. Lessne ${ }^{1,4}$ Kelvin Hong ${ }^{1}$ Anobel Tamrazi ${ }^{5}$
}

1Division of Vascular and Interventional Radiology, Johns Hopkins University School of Medicine, Baltimore, Maryland, United States

${ }^{2}$ Department of Radiology, University of Arkansas for Medical Sciences, Little Rock, Arkansas, United States

${ }^{3}$ Ciências Médicas de Minas Gerais, Belo Horizonte MG, Brazil

${ }^{4}$ Vascular and Interventional Specialists of Charlotte Radiology, Charlotte, North Carolina, United States

${ }^{5}$ Division of Vascular and Interventional Radiology, Palo Alto Medical Foundation
Address for correspondence Anobel Tamrazi, MD, PhD, Division of Vascular and Interventional Radiology, Palo Alto Medical Foundation, 2900 Whipple Avenue, Suite 225, Redwood City, CA 94062 (e-mail: atamrazi@gmail.com).

\begin{abstract}
Keywords

- pulmonary embolism

- thrombolysis

- pulmonary arterial pressure

The authors seek to evaluate hemodynamic parameters as potential clinical markers of real-time clinical improvement among patients with massive pulmonary embolism $(\mathrm{PE})$ in correlation with post-thrombolytic pulmonary arterial pressure improvement and overall clinical outcome. Thirteen patients with submassive or massive PE were admitted to the interventional radiology service and treated with catheter-directed thrombolysis. Among the four patients who qualified as massive PE, systolic blood pressure (BP) and vasopressor dependence suggested meaningful trends toward clinical improvement, after only $26.4 \%$ of treatment course/dose. Hemodynamic parameters such as systolic BP and inotropic vasopressor dependence may be considered in future treatment protocols as early indicators of treatment response.
\end{abstract}

\section{Introduction}

The pulmonary embolism (PE) spectrum of disease is the third most common cause of cardiovascular-related death in the United States. ${ }^{1}$ Massive pulmonary embolism (MPE) comprises approximately 5 to $10 \%$ of the PE disease spectrum, together with the more common submassive (SMPE) and nonmassive counterparts. ${ }^{2}$ MPE results in a disproportionately high degree of patient PE-related mortality, with an associated in-hospital mortality of $15 \%{ }^{3}$ Peripheral intravenous (IV) thrombolytic therapy has been the standard of care for several years ${ }^{4}$; however, there might be a considerable clinical reluctance in its common use, predominantly based on the concerns of posttreatment iatrogenic hemorrhage. As such, most MPE patients have remained on conservative treatment regimens, despite their more guarded prognosis.

There has been a recent regrowth of interest in chemical thrombolysis, with newer focus on catheter-directed thrombolysis (CDT), after the development and refinement of newer thrombolytic agents relative to those used several decades ago. ${ }^{5,6}$ The considerable bleeding risks might be, at least in part, mitigated by the substantially lower doses that can be administered locally within the pulmonary artery via CDT. Additionally, recent trials with infusion catheters using ultrasound-assisted mechanical thrombolysis along with chemical thrombolysis have demonstrated improvement in complications rates relative to peripheral IV chemical thrombolysis alone. ${ }^{7,8}$

Hypotension and systemic end-organ hypoperfusion are typical clinical manifestations of MPE. These patients typically necessitate dynamic treatment, often in an intensive care unit (ICU), with hour-by-hour monitoring of status. These patients are monitored in the real time with numerous clinical parameters, including, but not limited to, peripheral arterial blood pressure (BP), heart rate (HR), oxygen $\left(\mathrm{O}_{2}\right)$ saturation, $\mathrm{O}_{2}$ rate, and inotropic pressor dose (when used). In this study, the authors attempt to identify the potential clinical markers related to real-time hemodynamic status of the patient, which correlate with early posttreatment clinical received

July 29, 2017

accepted after revision

January 9, 2018
DOI https://doi.org/

10.1055/s-0038-1642108.

ISSN 2457-0214.
Copyright $\odot 2018$ by Indian Society of Vascular and Interventional Radiology
License terms

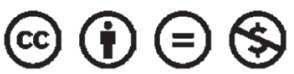


improvement, including reduction in pulmonary arterial pressure (PAP), to quantify the time at which the patients exhibit persistent clinical improvement during MPE CDT. The authors postulate that the same MPE disease parameters that define it along the PE spectrum (hypotension and/or inotropic vasopressor dependence) and confer its prognostic value relative to lower risk PE subgroups could be used as real-time clinical markers of treatment efficacy.

\section{Materials and Methods}

Institutional review board approval was obtained for this HIPAA (Health Insurance Portability and Accountability Act of 1996)-compliant retrospective case series. Records of PE patients treated by the interventional radiology service with CDT were analyzed for a consecutive 5-month period from April through August of 2014. Thirteen patients (6 men and 7 women; age range: 36-79; mean: 54.4 years) were stratified by PE subgroup classification into massive (MPE) or submassive (SBPE) groups. Distinction between MPE and SBPE was made on the basis of American Heart Association (AHA) criteria, which define that MPE has large central pulmonary arterial embolism, resulting in sustained hypotension (systolic blood pressure $[\mathrm{SBP}]<90 \mathrm{~mm} \mathrm{Hg}$ for at least 15 minutes or requiring inotropic support, not due to a cause other than $\mathrm{PE})$, pulselessness, or persistent profound bradycardia. ${ }^{8}$

Among patients who met criteria for MPE, ongoing real-time hemodynamic parameters were retrospectively collected, including invasive and noninvasive peripheral arterial $\mathrm{BP}, \mathrm{HR}, \mathrm{O}_{2}$ saturation, $\mathrm{O}_{2}$ administration, and inotropic vasopressor dose (when used), during thrombolysis. Clinical improvement was defined by sustained improvement in systolic BP above $90 \mathrm{~mm} \mathrm{Hg}$ without recurrent drop below $90 \mathrm{~mm} \mathrm{Hg}$ (for $>15$ minute as defined by AHA criteria), or by sustained reduction in vasopressor dose, with a goal of systolic BP greater than $90 \mathrm{~mm} \mathrm{Hg}$. For other parameters, improvement was defined by continuous clinical progress such as downtrending tachycardia, or decreasing $\mathrm{O}_{2}$ use. Patients were monitored throughout their infusion period in an ICU setting. Preprocedure main PA pressures were obtained via catheter at the time of treatment initiation, as well as posttreatment immediately before the catheter(s) were withdrawn at the conclusion of CDT. Posttreatment PAP improvement was correlated with time course of systolic BP improvement and vasopressor dose reduction. All patients were treated with either unilateral or bilateral 6F EKOS infusion catheters (EKOS Corporation, Bothell, Washington, United States) via internal jugular (IJ) vein access in the right or left pulmonary arteries. Alteplase was the sole thrombolytic agent used among all patients.

The technique for CDT is based on venous access through the IJ vein with one or two separate short $6 \mathrm{~F}$ sheaths, depending on need for unilateral versus bilateral catheters needed for that patient. After venous access, the right and left pulmonary arteries were accessed using standard catheter and wire techniques under fluoroscopic guidance. A limited hand injection was performed to confirm proper location. Initial pressures were measured using the catheter access within the main, right, or left pulmonary artery. Then over the wire, a 5.4F $106 \mathrm{~cm}$ EKOS (BTG) catheter with a $12-\mathrm{cm}$ infusion length was placed either in the right or left pulmonary artery and secured in place. No thrombectomy devices were used. The infusion of the tissue plasminogen activator (tPA) was then initiated as described for each patient via the EKOS infusion catheter. The patients with EKOS infusion catheters were all monitored in the ICU setting with critical care nursing staff. At the conclusion of the infusion, the pressures were induced at bedside in the ICU via the EKOS catheters prior to removal of the catheters at bedside. The IJ sheaths were then removed and hemostasis was achieved with manual compression.

\section{Results}

Among the four patients with MPE (-Table 1), two qualified on the basis of hypotension alone, zero qualified on the basis of inotropic vasopressor dependence alone, and two qualified on the basis of a combination of both. Among the four patients, there was a $46.3 \%$ average reduction in PAP noted over the treatment course ( - Table 2 ), with CDT treatment ranging from 9.75 to 24 hours, (mean $=18.8$ hours) (-Figs. 1-4). Clinical parameters, including $\mathrm{HR}, \mathrm{O}_{2}$ saturation, and $\mathrm{O}_{2}$ administration demonstrated no meaningful trend to suggest improvement (data not shown). For example, the authors noted that HR monitoring in the four patients did not correlate with clinical improvement and treatment efficacy. HR is an example of a nonideal clinical biomarker most likely due to the sensitive and variable nature of the patient's heart rate with vasopressors, volume status, and associated discomfort in a monitored unit. On the other hand, the four patients exhibited meaningful clinical improvement in both SBP and vasopressor dependence. These improvements were recognized

Table 1 Treatment data regarding the four treated MPE patients

\begin{tabular}{|c|c|c|c|c|c|c|}
\hline Patient & $\begin{array}{l}\text { Total } \\
\text { time (h) }\end{array}$ & $\begin{array}{l}\text { Rate } \\
\text { (mg/h/cath) }\end{array}$ & $\begin{array}{l}\text { Total tPA } \\
\text { dose }(\mathrm{mg})\end{array}$ & $\begin{array}{l}\text { Number of } \\
\text { catheters }\end{array}$ & $\begin{array}{l}\text { Initial SBP } \\
<90 \mathrm{~mm} \mathrm{Hg} \times 15 \mathrm{~min}\end{array}$ & $\begin{array}{l}\text { Pressor } \\
\text { dependent }\end{array}$ \\
\hline Patient A & 20 & 0.5 & 20 & 2 & Yes & Yes \\
\hline Patient B & 22 & 1 & 22 & 1 & Yes & No \\
\hline Patient C & 9.75 & 1 & 19.5 & 2 & Yes & Yes \\
\hline Patient D & 24 & 0.5 & 24 & 2 & Yes & No \\
\hline Average & 18.8 & 0.75 & 21.4 & - & - & - \\
\hline
\end{tabular}

Abbreviations: MPE, massive pulmonary embolism; SBP, systolic blood pressure; tPA, tissue plasminogen activator. 
Table 2 Catheter-based systolic PAP values and improvements seen among the four treated MPE patients

\begin{tabular}{|l|l|l|l|}
\hline & \multicolumn{1}{|l|}{$\begin{array}{l}\text { Systolic PAP at time of treatment } \\
\text { initiation (mm Hg) }\end{array}$} & \multicolumn{1}{l|}{$\begin{array}{l}\text { Systolic PAP at time of treatment } \\
\text { completion (mm Hg) }\end{array}$} & \multicolumn{2}{l|}{$\begin{array}{l}\text { Improvement in } \\
\text { systolic PAP (\%) }\end{array}$} \\
\hline Patient A & 46 & 16 & 55.2 \\
\hline Patient B & 44 & 20 & $54.5 \%$ \\
\hline Patient C & 60 & 27 & 55.0 \\
\hline Patient D & 72 & 56 & 22.2 \\
\hline Average & 55.5 & 29.8 & 46.3 \\
\hline
\end{tabular}

Abbreviations: MPE, massive pulmonary embolism; PAP, pulmonary arterial pressure.
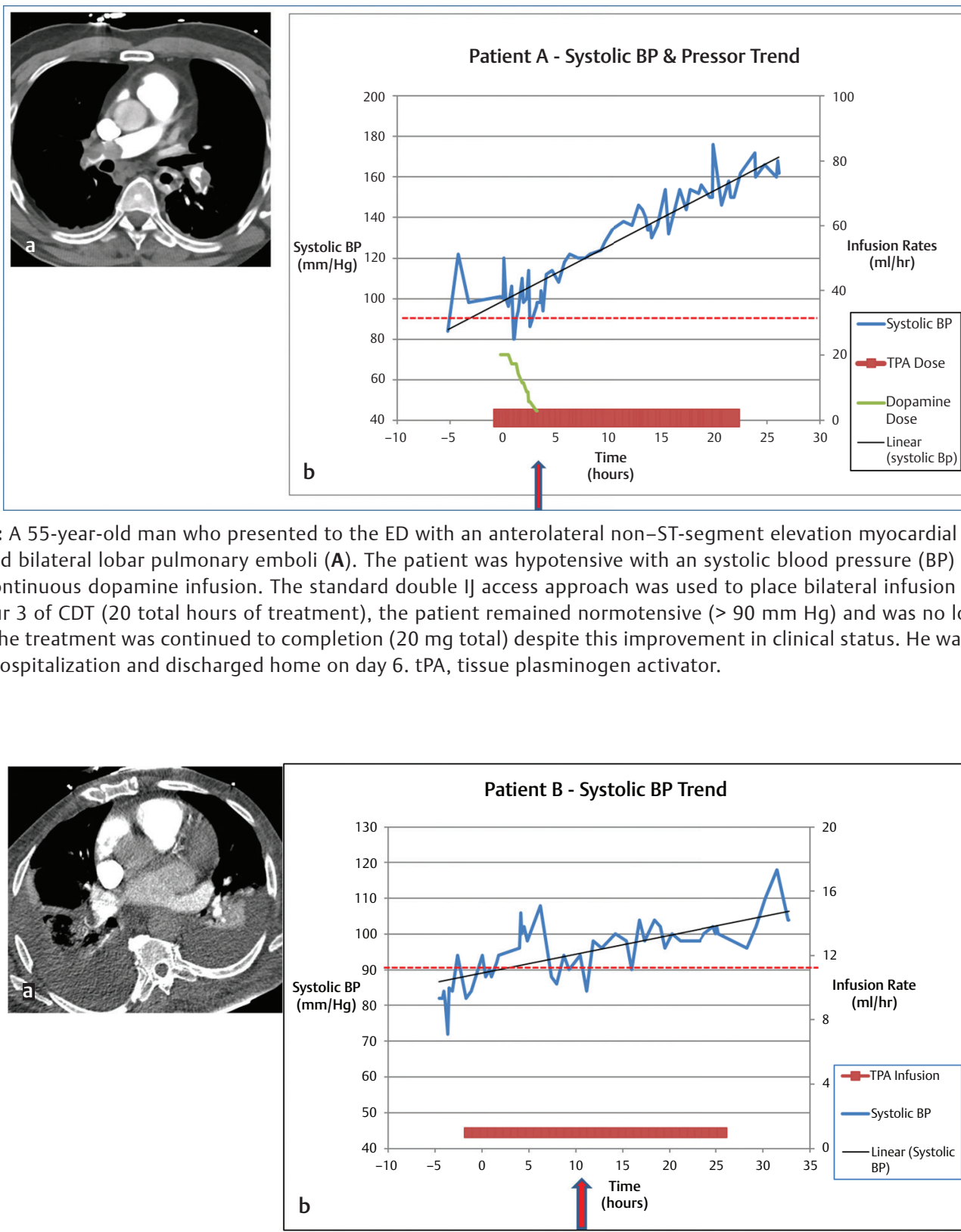

Fig. 2 Patient B: A 78-year-old man who was found on the ground with new atrial fibrillation. CTA in the ED revealed large right lobar and smaller left segmental pulmonary emboli (A). The patient was markedly hypotensive, with a systolic blood pressure (BP) as low as $74 \mathrm{~mm} \mathrm{Hg}$ but was not vasopressor dependent. The standard II access approach was used to place a unilateral right infusion catheter and initiate CDT. By hour 10 of CDT (22 total hours of treatment), the patient remained normotensive without need for any vasopressors (B). The treatment was continued to completion ( $22 \mathrm{mg}$ total) despite this improvement in clinical status. The patient was downgraded from ICU on hospital day 2 and discharged from hospital on day 20, with delay in discharge related to social and medical management of numerous underlying comorbidities. tPA, tissue plasminogen activator. 

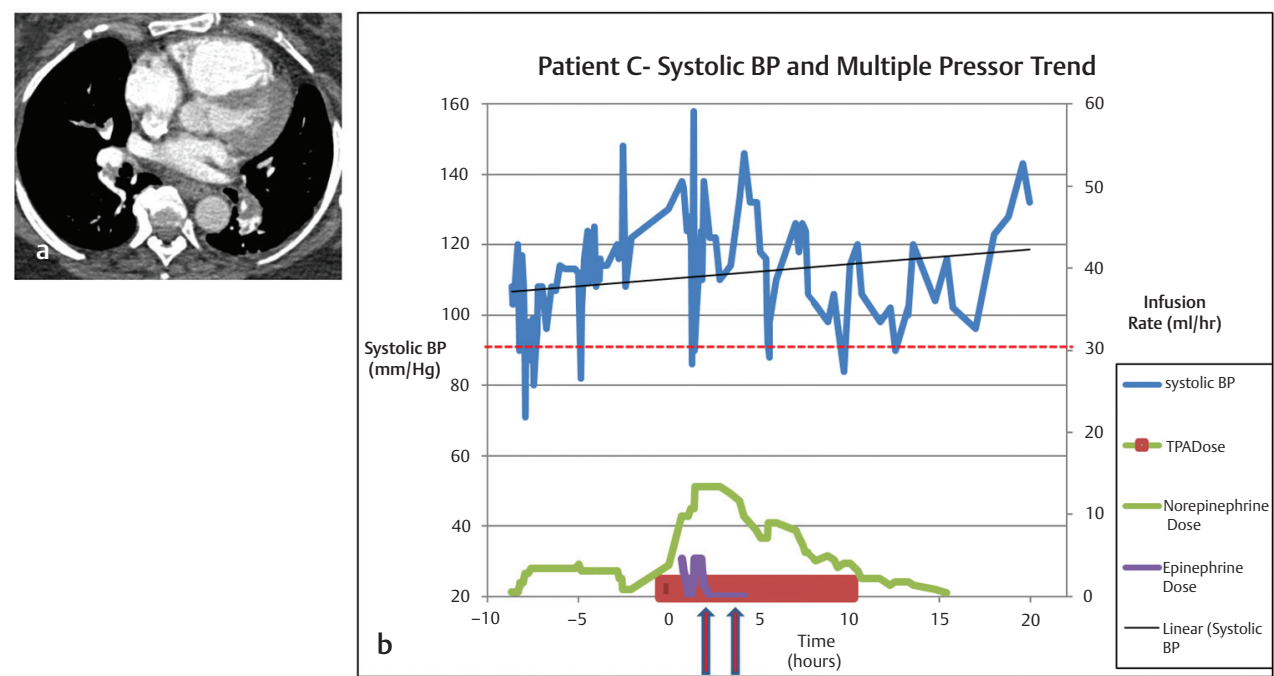

Fig. 3 Patient C: A 58-year-old woman who presented to the ED in PEA arrest. CT revealed large bilateral central PE (A). She qualified for MPE on the basis of both hypotension and vasopressor dependence. She necessitated continuous infusion epinephrine along with superimposed pulse doses of norepinephrine. The standard double IJ access approach was used to place bilateral infusion catheters and initiate CDT. By hour 2.5 of CDT (19.5 total hours of treatment), norepinephrine doses were no longer needed, and by hour 4 (9.75 hours of total treatment), epinephrine dose was downtrending. The patient's systolic blood pressure (BP) never returned below $90 \mathrm{~mm} \mathrm{Hg}$ for $>15$ minutes (AHA criteria) throughout remainder of treatment course (B). The treatment was continued to completion (19.5 mg total) despite this improvement in clinical status. She was downgraded from the ICU on hospital day 3 and discharged on hospital day 5. tPA, tissue plasminogen activator.
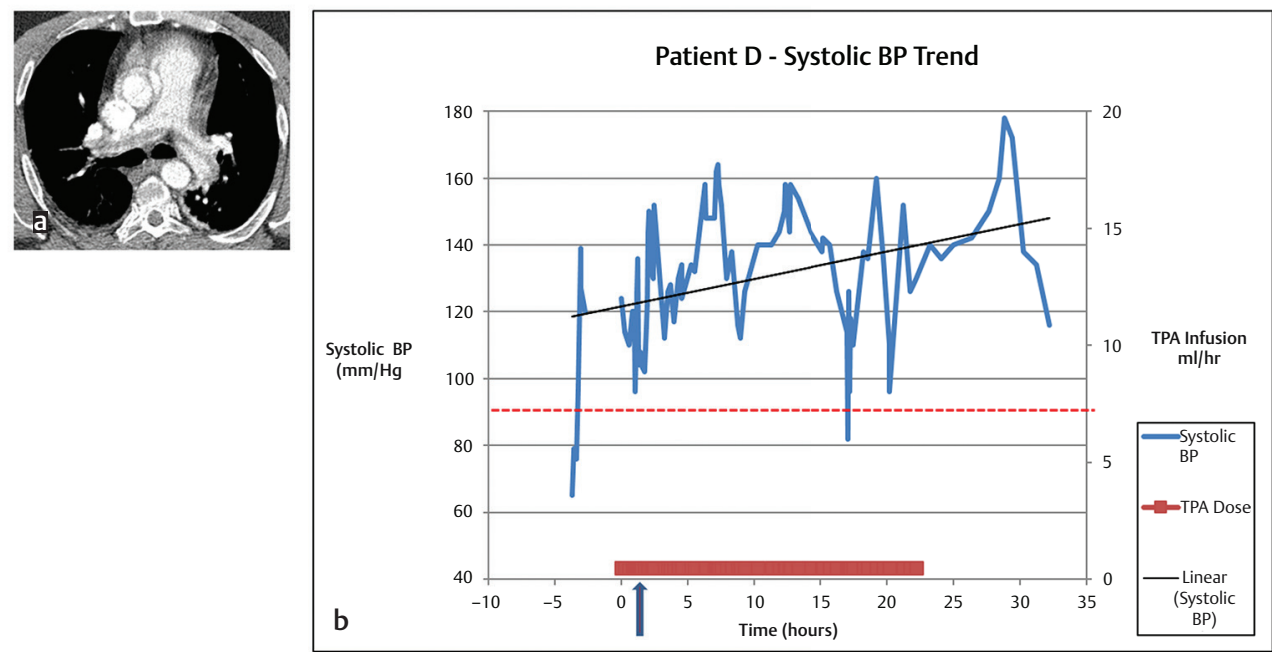

Fig. 4 Patient D: A 43-year-old man who was located in the hospital for multiple congenital neurologic issues and developed worsening hypoxia. CT revealed large central bilateral PE (A). The patient was markedly hypotensive with systolic blood pressure (BP) in 60s. The standard double IJ access approach was used to place bilateral infusion catheters and initiate CDT. She improved nearly immediately after infusion of tissue plasminogen activator (tPA). Systolic BP was initially labile during treatment, but continuous uptrend in SBP was noted by hour 3 of CDT (24 total hours of treatment) (B). The treatment was continued to completion ( $24.0 \mathrm{mg}$ total) despite this improvement in clinical status. He was discharged from ICU on day 13, due to neurologic complications, and discharged from hospital on days 26 after multiple secondary illnesses including hospital-acquired pneumonia.

by hours $3,10,4$, and 3 (mean $=5$ hours), among the four patients respectively (-Figs. 1-4), as earlier. This early 5-hour clinical improvement, noted in the setting of an 18.8-hour average CDT treatment course, represents substantial clinical improvement after only $26.4 \%$ of total CDT treatment time and dose.

\section{Discussion}

Massive pulmonary embolism has been proven to be a more labile and severe subset of PE than its SMPE counterpart.
Although thrombolysis is considered reasonable in patients with acute massive PE, ${ }^{9}$ the risk of post-treatment hemorrhage does remain a concern, with one meta-analysis documenting the risk of major bleeding associated with IV thrombolysis to be $9.2 \%$ versus $3.4 \%$ among a similar cohort treated with heparin alone. ${ }^{10}$ Superimposed concerns exist regarding the 1.5 to $3 \%$ incidence of intracranial bleeding, which has been often recorded in the literature. ${ }^{11}$ Early data released in two CDT thrombolysis trials ${ }^{7,8}$ have shown considerable promise with this therapy. 

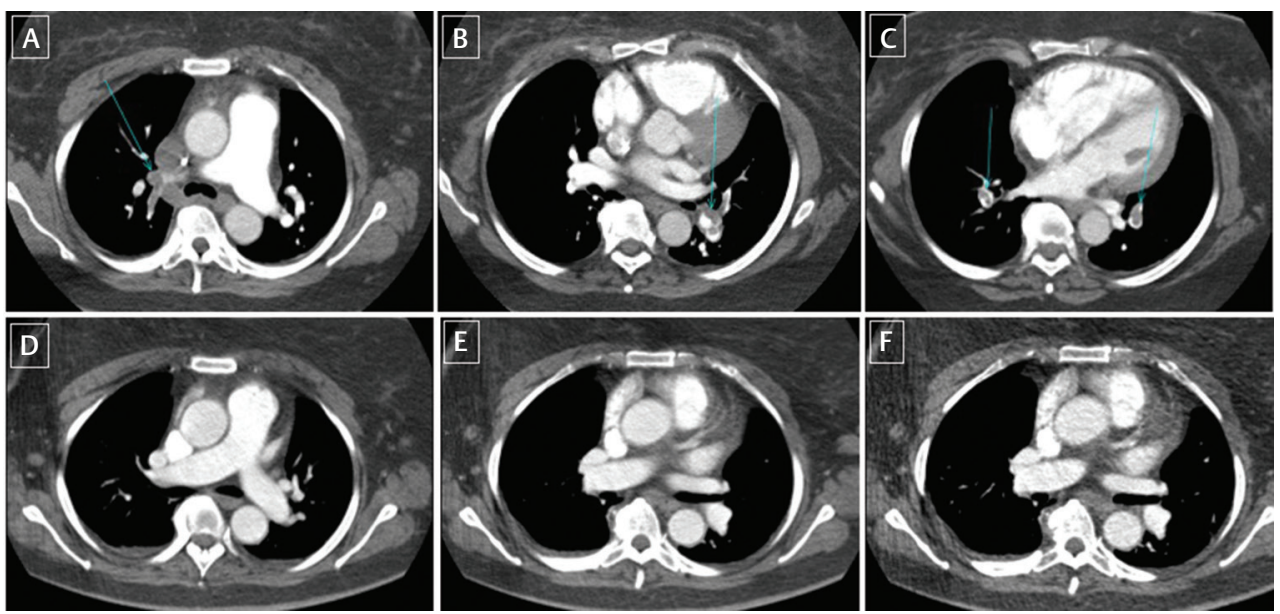

Fig. 5 Representative initial (A-C) and 2-month follow-up (D-F) axial CT images for patient A. The initial CT images demonstrated large bilateral lobar PEs (arrows in A-C). The follow-up CT done at 2 months shows complete resolution of the emboli (D-F).

In addition to improved patient safety/bleeding profiles, they have documented early reduction in right ventricular/left ventricular $(\mathrm{RV} / \mathrm{LV})$ ratios, a frequently used imaging parameter in both MPE and SMPE patients as a marker of disease severity, and early success, 24 to 48 hours posttreatment.

Even when chemical thrombolysis is selected as the treatment choice for this dynamic subset of disease (MPE), patients traditionally have been treated with fixed dose alteplase or tPA protocols. It would seem intuitive that the same conventional diagnostic parameters set forth by the AHA to define MPE, namely hemodynamic instability and vasopressor dependence, and which confer significant prognostic value to the patient, could be used in real time to ascertain improvement in patient status. This MPE experience demonstrates an improvement in BP and vasopressor dependence, within an average of 5 hours into an average treatment course of 18.8 hours, only $26.4 \%$ of the time course through their completed treatment. This early improvement correlates with an average $46.3 \%$ reduction in PAP, which took place directly over the course of treatment.

The prospect for real-time markers/metrics is of significant value in the care of the MPE patient, in several different capacities. Several studies ${ }^{12}$ in the past decade have reflected upon the dose-dependent risk associated with systemic chemical thrombolysis. Lower-dose protocols have yielded improved bleeding profiles. Furthering this argument, the data available from the recent ULTIMA and SEATTLE II trials have again demonstrated that lower doses (typically $24 \mathrm{mg}$ of alteplase over the course of 24 hours) again confer improved bleeding profiles. This finding was again demonstrated among the relatively small patient population of this study, with no major or minor bleeding encountered among the four MPE patients who received an average of $21.5 \mathrm{mg}$ of alteplase over an average of 18.8 hours. Parenthetically, this was also observed among the nine SBPE patients as well who had no major bleeding events reported.

If thrombolysis can be monitored, or at least broadly quantified in the real-time setting, doses could potentially be modulated and decreased among early responders, or conversely, increased in those refractory to early therapy or those who exhibit early clinical decline. Critically ill MPE patients at high risk for major bleeding (i.e., recent intracranial hemorrhage) $)^{13}$ could potentially be treated for mere mitigation of hypotension and shock rather than through a full-fixed treatment protocol. Future interest lies in further validation of these hemodynamic markers as well as evaluation of new markers among, which may include mixed venous $\mathrm{O}_{2}$ saturation, to better evaluate for progressively improving tissue perfusion and oxygenation, as well as real-time main PAP to assess for improving pulmonary vasculature hemodynamics and flow.

This study has several limitations. First, the small sample size of four MPE patients may be insufficient to definitively correlate with the clinical implications of these markers, which remains a subject of future research. Second, the authors did not have a "negative control" among treatment nonresponders, as all of the patients in this cohort clinically improved. Third, the SMPE subgroup was not further analyzed because the focus of this study was MPE patients. Lastly, there may be other clinically useful markers of treatment response, which were not studied in this report.

In conclusion, in MPE patients undergoing CDT, hemodynamic parameters such as SBP and inotropic vasopressor dose may be considered in future treatment protocols as early indicators of treatment response.

\section{Disclosures}

The authors have no disclosures to declare.

\section{References}

1 Glynn RJ, Danielson E, Fonseca FA, et al. A randomized trial of rosuvastatin in the prevention of venous thromboembolism. N Engl J Med 2009;360(18):1851-1861

2 Kucher N, Rossi E, De Rosa M, Goldhaber SZ. Prognostic role of echocardiography among patients with acute pulmonary embolism and a systolic arterial pressure of $90 \mathrm{~mm} \mathrm{Hg}$ or higher. Arch Intern Med 2005;165(15):1777-1781

3 Kucher N, Rossi E, De Rosa M, Goldhaber SZ. Massive pulmonary embolism. Circulation 2006;113(4):577-582

4 Kearon C, Akl EA, Ornelas J, et al. Antithrombotic therapy for VTE disease: CHEST guideline and expert panel report. Chest 2016;149(2):315-352 
5 Leeper KV Jr, Popovich J Jr, Lesser BA, et al. Treatment of massive acute pulmonary embolism. The use of low doses of intrapulmonary arterial streptokinase combined with full doses of systemic heparin. Chest 1988;93(2):234-240

6 Vujic I, Young JW, Gobien RP, Dawson WT, Liebscher L, Shelley BE Jr. Massive pulmonary embolism: treatment with full heparinization and topical low-dose streptokinase. Radiology 1983;148(3):671-675

7 Kucher N, Boekstegers P, Müller OJ, et al. Randomized, controlled trial of ultrasound-assisted catheter-directed thrombolysis for acute intermediate-risk pulmonary embolism. Circulation 2014;129(4):479-486

8 Piazza G, Hohlfelder B, Jaff MR, et al; SEATTLE II Investigators. A prospective, single-arm, multicenter trial of ultrasoundfacilitated, catheter-directed, low-dose fibrinolysis for acute massive and submassive pulmonary embolism: the SEATTLE II study. JACC Cardiovasc Interv 2015;8(10):1382-1392

9 Jaff MR, McMurtry MS, Archer SL, et al; American Heart Association Council on Cardiopulmonary, Critical Care, Perioperative and Resuscitation; American Heart Association Council on Peripheral Vascular Disease; American Heart
Association Council on Arteriosclerosis, Thrombosis and Vascular Biology. Management of massive and submassive pulmonary embolism, iliofemoral deep vein thrombosis, and chronic thromboembolic pulmonary hypertension: a scientific statement from the American Heart Association. Circulation 2011;123(16):1788-1830

10 Chatterjee S, Chakraborty A, Weinberg I, et al. Thrombolysis for pulmonary embolism and risk of all-cause mortality, major bleeding, and intracranial hemorrhage: a meta-analysis. JAMA 2014;311(23):2414-2421

11 Meyer G, Vicaut E, Danays T, et al; PEITHO Investigators. Fibrinolysis for patients with intermediate-risk pulmonary embolism. N Engl J Med 2014;370(15):1402-1411

12 Sharifi M, Bay C, Skrocki L, Rahimi F, Mehdipour M; "MOPETT" Investigators. Moderate pulmonary embolism treated with thrombolysis (from the "MOPETT" Trial) Am J Cardiol 2013;111(2):273-277

13 Bottinor W, Turlington J, Raza S, et al. Life-saving systemic thrombolysis in a patient with massive pulmonary embolism and a recent hemorrhagic cerebrovascular accident. Tex Heart Inst J 2014;41(2):174-176 